\title{
LICORICE TRITERPENEACIDS AS SCAFFOLDS FOR OBTAINING NEW INHIBITORS OF FLAVIVIRUSES
}

\author{
L. Baltina', Cheng-Wen Lin ${ }^{2}$ \\ ${ }^{1}$ Ufa Institute of Chemistry, Ufa Federal Research Centre of RAS, \\ 450054, Russia, Ufa, Prospect Oktyabrya,71. \\ ${ }^{2}$ Departmentof Medical Laboratory Science and Biotechnology, China Medical University, \\ 40402, Taiwan, Taichung, Hsueh-Shih Rd.,91.
}

DOI: 10.19163/MedChemRussia2021-2021-436

E-mail: baltina@abrb.ru

Glycyrrhizic acid (GA) (1) and its aglycone - glycyrrhetic acid (GLA) (2), the main components of licorice root (Glycyrrhiza glabra L.), are among the available triterpene acids with a wide spectrum of biological activity, including immunotropic and antiviral [1]. This work is devoted to the synthesis of libraries of GA and GLA derivatives in order to study them as inhibitors of the pathogenic flaviviruses Dengue and Zika, with the establishment of the structure-activity relationship and the identification of leading compounds.

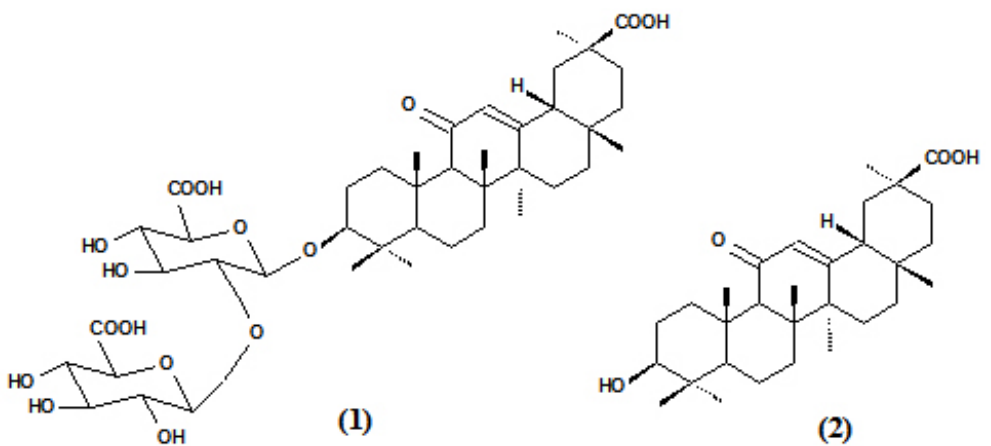

Modifications of GA and GLA were carried out to obtain new groups of nitrogen-containing derivatives (amino acid conjugates, acylhydrazones, amides, semi- and thiosemicarbazones, thiadiazoles and triazines). Mono- and disaccharide analogs of GA with a modified carbohydrate chain have been synthesized. As a result of in vitro screening of antiviral activity, among GA derivatives, effective inhibitors of Dengue 2 and Zika virus with IC50 $<1 \mu \mathrm{M}$ were found. GLA modification with the introduction of semicarbazide and thiadiazole pharmacophore groups is promising for the production of new low-toxic Zika virus inhibitors.

The research was carried out with the financial support of the RFBR and MOST with in the framework of scientific project No. 18-53-52004.

\section{References}

1. L.Baltina, R.Kondratenko, Current Bioactive Compounds, 2020, 16, 1; https://doi.org/10.2174/1573407216666200210122751. 